\title{
Apport du modèle géologique et géophysique 3D dans le développement du gisement polymétallique de Draa Sfar (Massif hercynien des Jebilet centrales, Maroc)
}

\author{
Contribution of geological and geophysical 3D models to the \\ development of the polymetallic deposit of Draa Sfar (Hercinian Massif \\ of Central Jebilets, Morocco)
}

\author{
S. Rziki ${ }^{1}$, A. Alansari ${ }^{2}$, EM. Mouguina ${ }^{2}$, J. Simard ${ }^{3}$, M. Zouhair ${ }^{4}$, L. Maacha ${ }^{5}$
}

RESUME

La compilation des données géologiques et géophysiques (magnétique et gravimétrique) et l'élaboration des modèles tridimensionnels correspondants ont démontré l'enracinement des minéralisations qui a permis la remise en activité de la mine Draa Sfar après 23 ans d'arrêt. Les ressources géologiques ont été multipliées dix fois en trois années pour atteindre $10 \mathrm{Mt}$ à $5,39 \% \mathrm{Zn} 1,94 \% \mathrm{~Pb}$ et $0,34 \% \mathrm{Cu}$. Cette méthodologie d'exploration basée sur les modèles géologique et géophysique tridimensionnels devient de plus en plus utilisée dans l'exploration minière stratégique et tactique en raison, entre autres, des avancées en informatique et de la disponibilité d'outils de modélisation spécialisés. Elle pourrait être d'un grand secours pour le développement minier de la province des amas sulfurés des Jebilet de Guemassa et par extension à d'autres gîtes présentant des caractéristiques géologiques et métallogéniques similaires.

Mots-clés: Draa Sfar, Amas sulfurés, Polymétallique, Hercynien, Modèle géologique et géophysique 3D, Jebilet, Maroc.

\begin{abstract}
The compilation of geological and geophysical (magnetic and gravity) data and the development of the corresponding three-dimensional models have allowed the recognition of the deep mineralized structures, and helped the reopening of the mining after 23 years of inactivity. Geological resources were increased tenfold in three years to reach $10 \mathrm{Mt}$ at $5.39 \% \mathrm{Zn}, 1.94 \% \mathrm{~Pb}$ and $0.34 \% \mathrm{Cu}$.

This exploration methodology based on three-dimensional geological and geophysical models becomes increasingly used in mining exploration strategy after the improvement in the computing capacities of the modelling tools. The results from this study could be helpful for the development of mining massive sulphide deposits in the Jebilet province of Guemassa and by extension to other deposits with similar geological and metallogenic characteristics.
\end{abstract}

Keywords: Draa Sfar, Massif sulphide, Polymetallic, Hercynian, 3D Model geophysical and geological, Jebilet, Morocco

1 Cadre géotechnicien au Site de Draa Sfar, BP 469, Marrakech, Maroc. Email: rziki@managem-ona.com

2 PES, Université Cadi ayyad, Faculté des Sciences Semlalia, BP 2390, Marrakech, Maroc.

3 Directeur de Sagax Maghreb, Marrakech, Maroc.

4 Directeur exploration Maroc à Managem, Twin center, tour A BP5199, Casablanca, Maroc.

5 Directeur générale exploration et développement à Managem, Twin center, tour A BP5199, Casablanca, Maroc. 


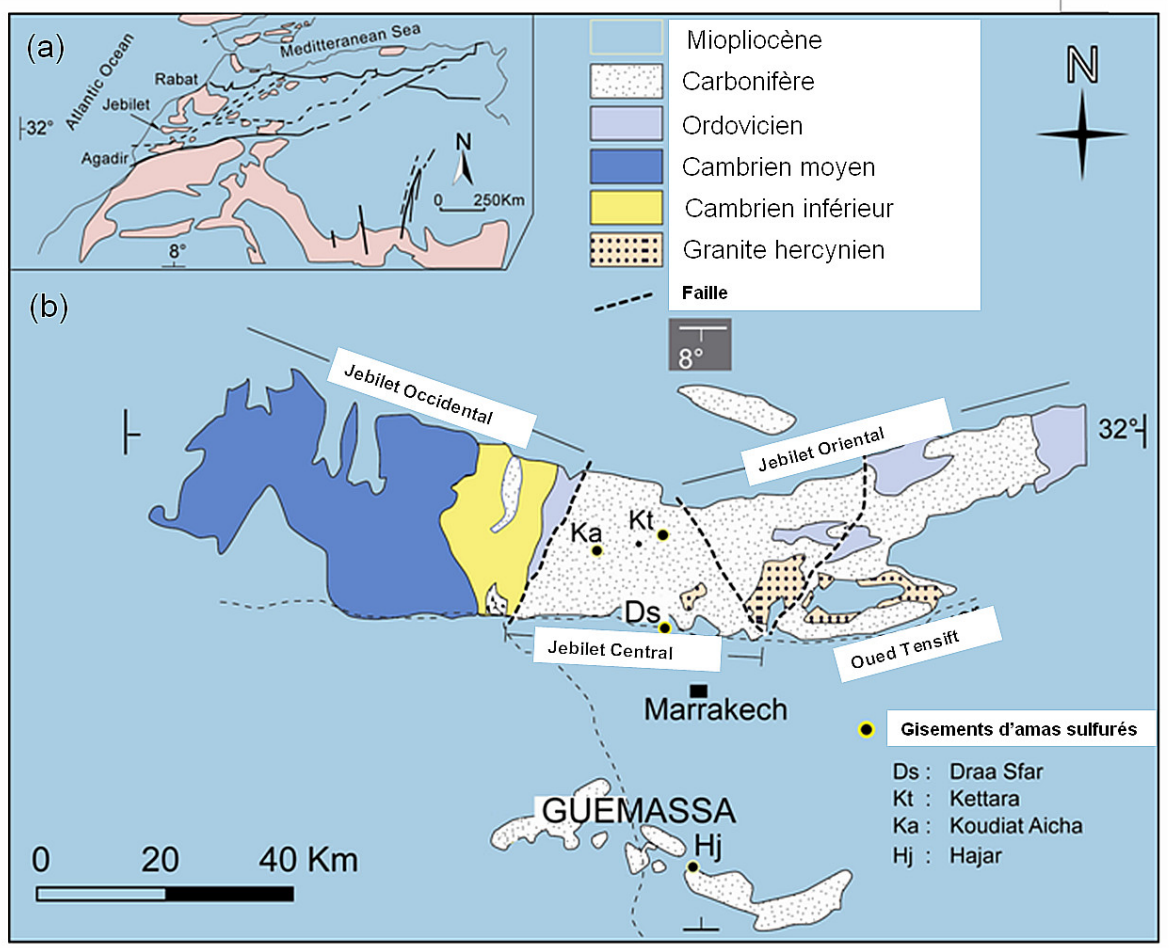

Fig. 1.-(a) Localisation du massif des Jebilet en Afrique du nord. (b) Carte géologique générale des affleurements hercyniens des Jebilet- Guemassa avec la localisation des principaux gisements d'amas sulfurés, d'après Huvelin 1977, modifiée.

\section{Introduction}

Le gisement polymétallique à zinc, plomb et cuivre de Draa Sfar situé à $13 \mathrm{Km}$ au NW de la ville de Marrakech (massif hercynien des Jebilet), a été découvert en 1953. Par la suite, cette découverte a été confirmée en 1962 par une anomalie magnétique au sol (Huvelin, 1977).

Une première phase d'exploitation basée sur l'interprétation de cette anomalie magnétique a été arrêtée après trois années d'exploitation en raison de l'épuisement des ressources délimitées entre la surface et le niveau-110.

Récemment, l'exploitation du gisement a été reprise par une nouvelle compagnie, après 23 ans d'arrêt, suite à l'interprétation des nouvelles données géologiques et géophysiques ayant démontrés l'enracinement des corps minéralisés.

Cette publication a pour but de présenter la méthodologie d'exploration qui été appliquée pour le développement du gisement de Draa Sfar et que nous recommandons pour des gisements présentant des caractéristiques similaires au Maroc et ailleurs comme le gisement de Transfiguration au Canada (Bédart et al., 2006).

\section{Contexte géologique et métallogénique}

Le gisement de Draa Sfar appartient à la province métallogénique des amas sulfurés à pyrrhotite dominante du massif hercynien des Jebilet- Guemassa (Huvelin, 1977; Bernard et al., 1988; Souaré,1988; Ben aissi, 2001; Hibti, 2001; Rziki, 2006; Belkabir et al., 2008; Moreno et al., 2008; Marcoux et al., 2008). Les corps minéralisés de Draa Sfar sont encaissés dans la série de Sarhlef qui couvre la majeure partie des Jebilet central (fig. 1). Cette série est composé d'une séquence sédimentaire gréso-pélitique (Huvelin, 1977; Bordonaro, 1983; Rziki, 2006; Moreno et al., 2008) avec des intercalations des roches volcaniques et volcanosédimentaires acides et basiques (Aarab,1995; Essaifi, 1995; Gibson et al., 2005 ). Récemment, une étude biostratigraphique réalisée sur l'encaissant de la minéralisation a permis d'attribuer un âge Asbien (Mississipien moyen) à la série de Sarhlef (Moreno et al., 2008). D'autres travaux ont porté sur l'étude des altérations hydrothermales associées à la minéralisation et sur la déformation ductiles des roches volcaniques (Ben aissi et al., 2005; Rziki, 2006; Belkabir et al., 2008). La datation 


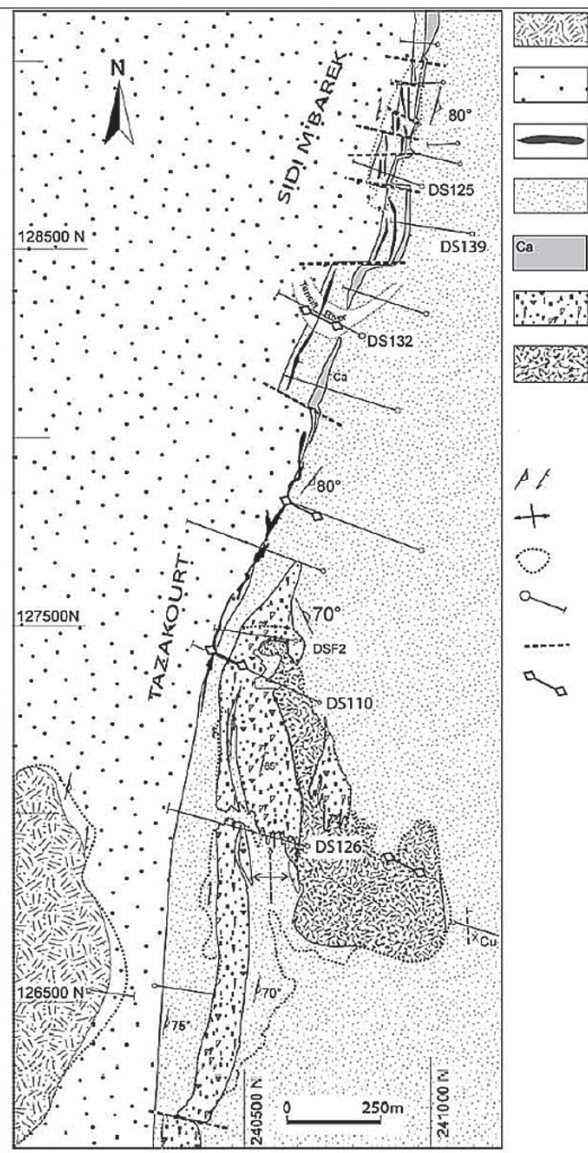

\section{Gabbro intrusif}

Métapélites noires carbonatées

Sulfures massifs

Métapélites gréseuses

Métapélites riches en bioclastes

Roches volcanoclastiques acides

Laves rhyodacitiques

Plans S0, S1

Axe d'anticlinal asymétrique

Limite des affleurements

Sondages carottés

Failles

Fig. 2.-Carte géologique de Draa Sfar montrant les affleurements de Tazakourt, les corps minéralisés reconnu au niveau-110 par traçage et les sondages carottés projetés.

$(\mathrm{U} / \mathrm{Pb})$ sur zircon des granodiorites des Jebilet a donné un âge de 330,5Ma (Essaifi et al., 2003) et la datation du cryptodôme rhyodacitique de Draa Sfar (fig. 2) par la méthode (Ar-Ar) des séricites a donné un âge de 331,7+/-7,9Ma (Marcoux et al., 2008). La série de Sarhlef est affectée par l'orogenèse hercynienne caractérisée par un métamorphisme de type schiste vert et un plissement synschisteux ayant succédé à la formation de plis d'axes subméridiens. Ce plissement majeur est suivi par des chevauchements, des cisaillements ductiles et des failles cassantes dont l'intensité et la géométrie sont variables (Huvelin, 1977; Bordonaro, 1983). Le gisement de Draa Sfar et les formations encaissantes ont subi l'effet d'une déformation ductile, hétérogène, non coaxiale, synschisteuse, régie par un fort serrage E-W qui se caractérise par la transposition de la stratification (S0) dans la schistosité de flux (S1) orienté $\mathrm{N} 10,75^{\circ} \mathrm{E}$ reprise par une schistosité de crénulation (S2) et un boudinage généralisé des formations compétentes. Les axes des boudins montrent un faible plongement vers le nord (Rziki, 2006).

\section{Les minéralisations}

\section{Morphologie du corps minéralisé}

Le gisement de Draa Sfar est de type amas sulfuré volcanogène (Huvelin, 1977; Bernard et al., 1988; Ben aissi, 2001; Hibti, 2001; Rziki, 2006; Belkabir et al., 2008; Moreno et al., 2008) étendu sur $1.5 \mathrm{Km}$ et découpé en trois bloc d'exploitation qui sont le bloc Sud, Central et Nord. La structure minéralisée est reconnue en surface par un chapeau de fer. L'amas est composé par deux structures minéralisées subverticales, espacées de $50 \mathrm{~m}$ (fig. 3). La première structure, cuprifère, distale et située à l'Est, est composée de pyrrhotite massive 
Section DSC1330N

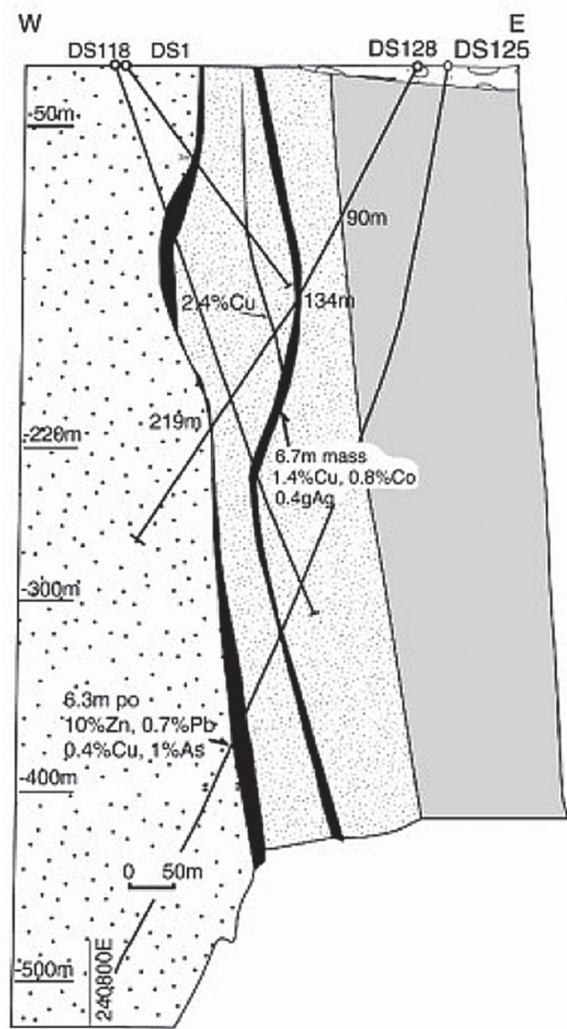

Section DSCOO

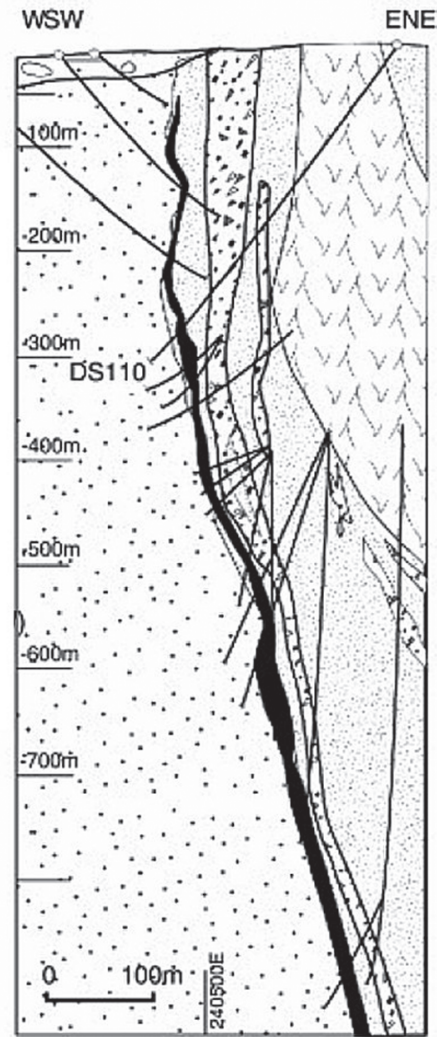

Fig. 3.-Coupes géologiques verticales passant par le sondage DS110 pour la coupe DSC00 et par le sondage DS125 pour la coupe DSC1330N.
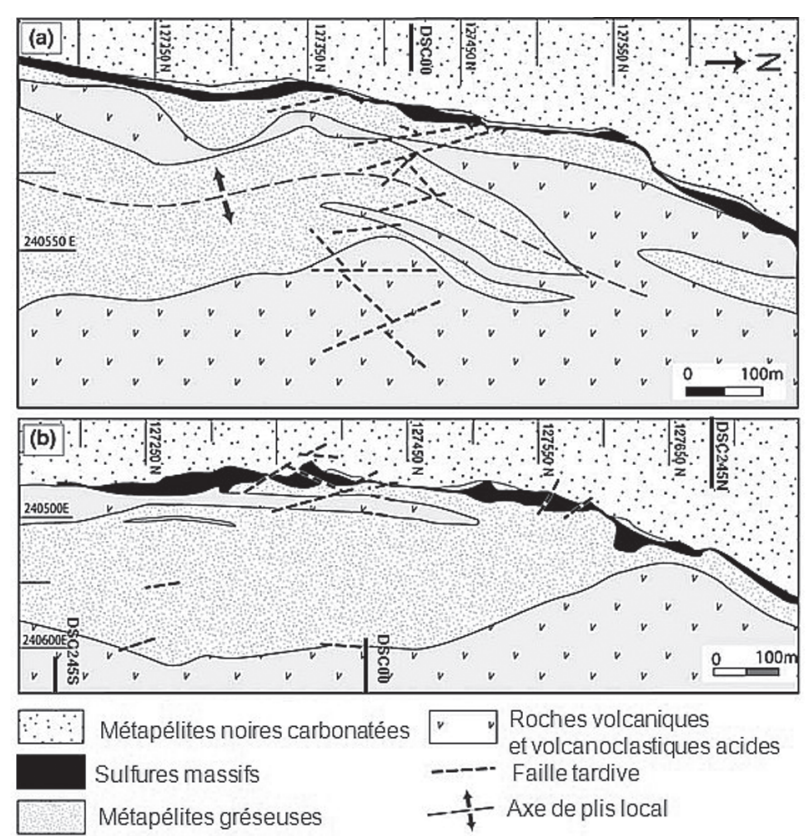

Fig. 4.-Plan géologiques simplifiés des premiers niveaux tracés à Draa Sfar (a) niveau $-300 \mathrm{~m}$ et (b) niveau $-400 \mathrm{~m}$. imprégnée de veines de chalcopyrite, avec un potentiel de $1.9 \mathrm{Mt}$ à $1 \% \mathrm{Cu}$. La deuxième structure, zincifère et située à l'ouest est plus importante, avec une puissance variable $(0,20 \mathrm{~m}$ à $30 \mathrm{~m})$. Elle est reconnue à coté des laves rhyodacitiques (fig. 4) jusqu'au niveau $-1000 \mathrm{~m}$, sous forme d'une méga lentille aplatie, boudinée et déversée vers l'ouest avec un fort pendage vers l'est. Les ressources délimitées dans la structure zincifère à la fin de 2009 totalisent $8.5 \mathrm{Mt}$ à $5,28 \% \mathrm{Zn}, 1,75 \% \mathrm{~Pb}$ et $0,35 \%$ $\mathrm{Cu}$. L'extrait géologique est de $2 \mathrm{Mt}$ tonnes à $6,15 \% \mathrm{Zn}, 2,66 \% \mathrm{~Pb}$ et $0,29 \% \mathrm{Cu}$.

\section{Minéralogie et succession paragénétique}

La quasi-totalité de la minéralisation en cours d'exploitation est un minerai massif, à texture rubanée, polymétallique, composé de pyrrhotite, sphalérite, galène, chalcopyrite, et traces d'arsénopyrite (Rziki, 2006). La chalcopyrite présente un caractère tardif en remplacement des pyrrhotites et des spha- 


\begin{tabular}{|c|c|c|c|}
\hline & $\begin{array}{c}\text { Stade } \\
\text { antécinématique }\end{array}$ & $\begin{array}{c}\text { Stade } \\
\text { syncinématique }\end{array}$ & $\begin{array}{c}\text { Stade } \\
\text { postcinematique }\end{array}$ \\
\hline Pyrrhotite & & & \\
\hline $\begin{array}{l}\text { Sphalérite } \\
\text { Arsénopyrite }\end{array}$ & & & \\
\hline $\begin{array}{l}\text { Galène } \\
\text { Chalcopvrite }\end{array}$ & & & - \\
\hline $\begin{array}{l}\text { Chalcopyrite } \\
\text { Pyrite }\end{array}$ & & & \\
\hline
\end{tabular}

Fig. 5.-Séquence paragénétique de la minéralisation sulfurée de Draa Sfar.

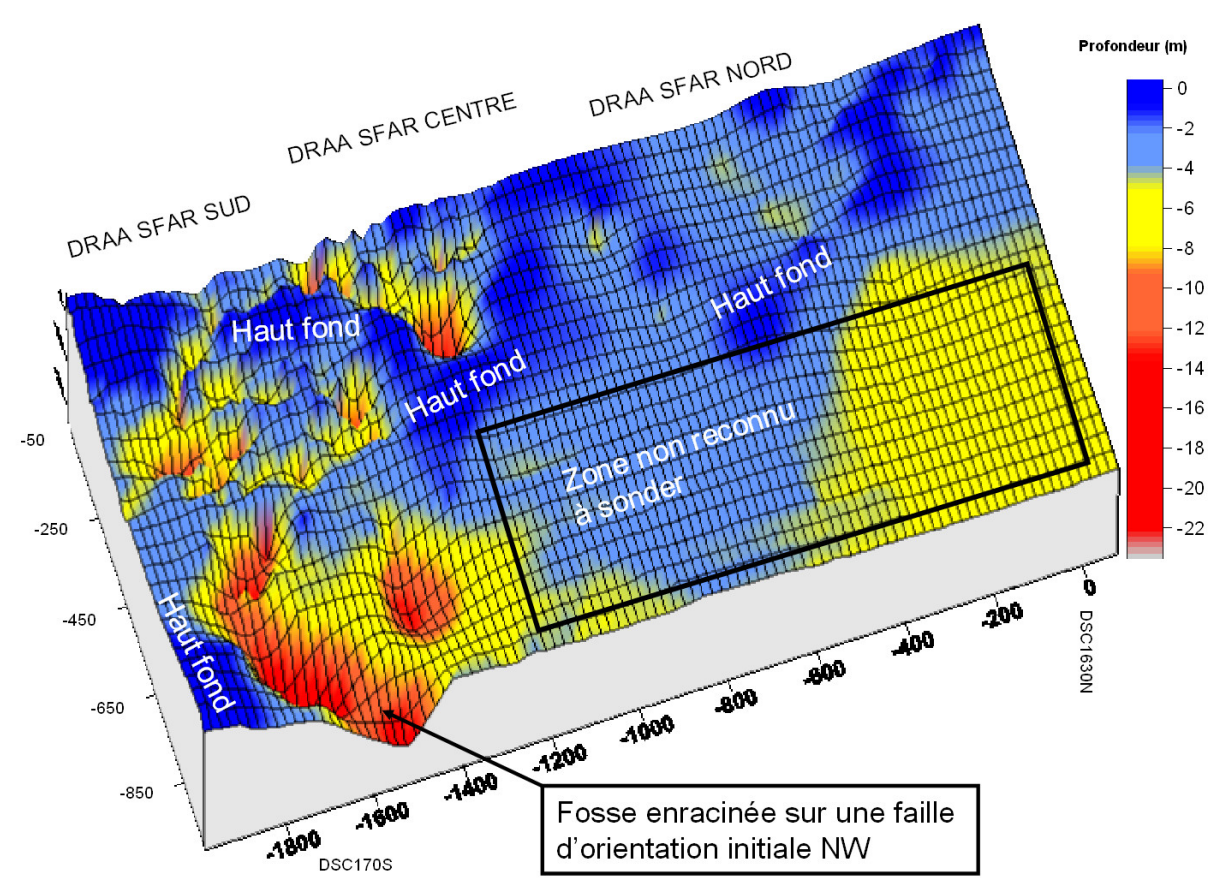

Fig. 6.-Modélisation tridimensionnelle des valeurs de la puissance horizontale misent en relief négatif permettant de visualiser la morphologie du mur de la minéralisation.

lérites qui ne subsistent parfois que sous forme de reliques squelettiques au sein de la chalcopyrite. Ce minerai présente des textures d'étirement de la sphalérite, de la pyrrhotite et de la chalcopyrite qui témoignent d'une déformation ductile (Hibti, 2001). Dans les zones épaisses on observe la dominance des textures de remplacement au mur de la minéralisation. Les relations texturales nous ont permis de conclure que la précipitation des cristaux d'arsénopyrite, de pyrrhotite, de sphalérite, de galène, de chalcopyrite et de pyrite s'est faite au cours d'une même phase minéralisatrice précoce. Cet assemblage a subi une recristallisation étagée avec un recouvrement de temps de cristallisation d'un minéral par rapport à l'autre (fig. 5).

\section{Reconstitutions des données géologiques en 3D}

Le modèle géologique 3D du gisement de Draa Sfar a été réalisé à l'aide des données géologiques provenant de la carte géologique, des levés des traçages $(5 \mathrm{Km})$ et des sondages carottés $(48 \mathrm{Km})$.

La première étape de modélisation avec le logiciel Vulcan de Maptek dont la mine détient trois licences (géologie, arpentage et mine), a consisté en la création d'une base de données sur le support MS-ACCESS, transférée sur le logiciel Vulcan. Ensuite, la fonction Batch-plotting du logiciel a été utilisée, pour l'établissement des coupes transver- 
sales espacées de $35 \mathrm{~m}$ et les plans de niveaux chaque $20 \mathrm{~m}$.

La procédure d'habillage d'une coupe géologique consiste à reporter d'une manière interprétative les structures géologiques en essayant d'interpoler les formes conformément aux effets de la tectonique. Après la digitalisation sur Vulcan des coupes et des plans habillés géologiquement, on a procédé à la construction des solides en 3D à partir des polygones. Le polygone correspondant à une surface donnée sur une section est discrétisé par une série de points. Deux polygones sur deux sections voisines sont joints par des triangles de façon à fermer le vide entre les sections. Le volume obtenu est découpé en blocs pour construire le modèle de blocs du gisement. A chaque bloc sont affectées des teneurs en métaux estimées à l'aide d'une méthode d'interpolation de type pondération par l'inverse du carré de la distance séparant le bloc et les échantillons autour et à l'intérieur d'un ellipsoïde de recherche de taille et d'orientation spécifiques (Dagbert et al., 2002).

Ce modèle a permis d'une part, l'estimation des ressources géologiques de chaque lentille en trois dimensions, la mise en évidence des axes de tendance des puissances horizontales de la structure minéralisée en 3D et de la zonalité des teneurs $(\mathrm{Zn}, \mathrm{Pb}, \mathrm{Cu})$ ainsi que leur relation spatiale avec les volcanites acides. D'autres parts, ce modèle a permis de tracer la paléogéographie du bassin de dépôt, qui montre l'existence des hauts fonds occupés par les laves massives et où la structure minéralisée est absente ou présente une faible puissance. De part et d'autre des hauts fonds, apparaissent des fosses d'une profondeur maximale de $-22 \mathrm{~m}$ (fig. 6).

\section{Présentation et interprétation des données géophysiques}

Les étapes suivies lors du traitement des données magnétiques et gravimétriques des différentes lentilles connues au niveau du gisement se présentent comme suit :

- Importation du modèle géologique 3D du gisement et des bases de données de susceptibilité magnétique et de densité.

- Calcul de la réponse magnétique et gravimétrique du gisement et des lithologies environnantes en fonction des propriétés physiques connues.

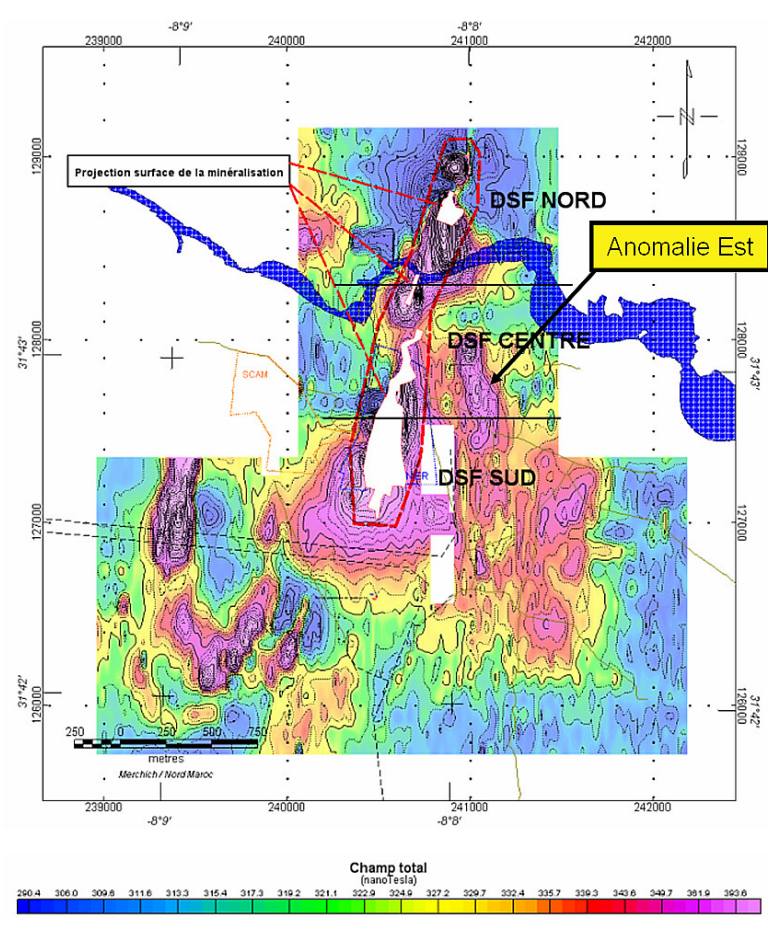

Fig. 7._Vue en plan du levé magnétique au sol, champ total.

- Adaptation des modèles théoriques magnétiques et gravimétriques jusqu'à l'obtention du meilleur ajustement possible entre réponses mesurées et théoriques.

- Examen des résultats obtenus et recommandations.

\section{Propriétés physiques}

Les valeurs de susceptibilité magnétique et de densité utilisées dans la modélisation sont issues des mesures sur les affleurements et sur quinze sondages mesurés tous les mètres avec un appareil de mesure de la susceptibilité magnétique modèle KT9 distribué par la firme Exploranium. Les résultats obtenus pour 20 échantillons de chaque faciès sont :

\begin{tabular}{lcc}
\hline Faciès & Susceptibilité (x10-3 SI) & Densité $(\mathrm{g} / \mathrm{cm} 3)$ \\
\hline Minerai & 100 & 4 \\
Tufs & 1,05 & 2,64 \\
Lave acide & 0,32 & 2,56 \\
Gabbro & 4 & 2,86 \\
Schiste & 0,25 & 2,55 \\
\hline
\end{tabular}




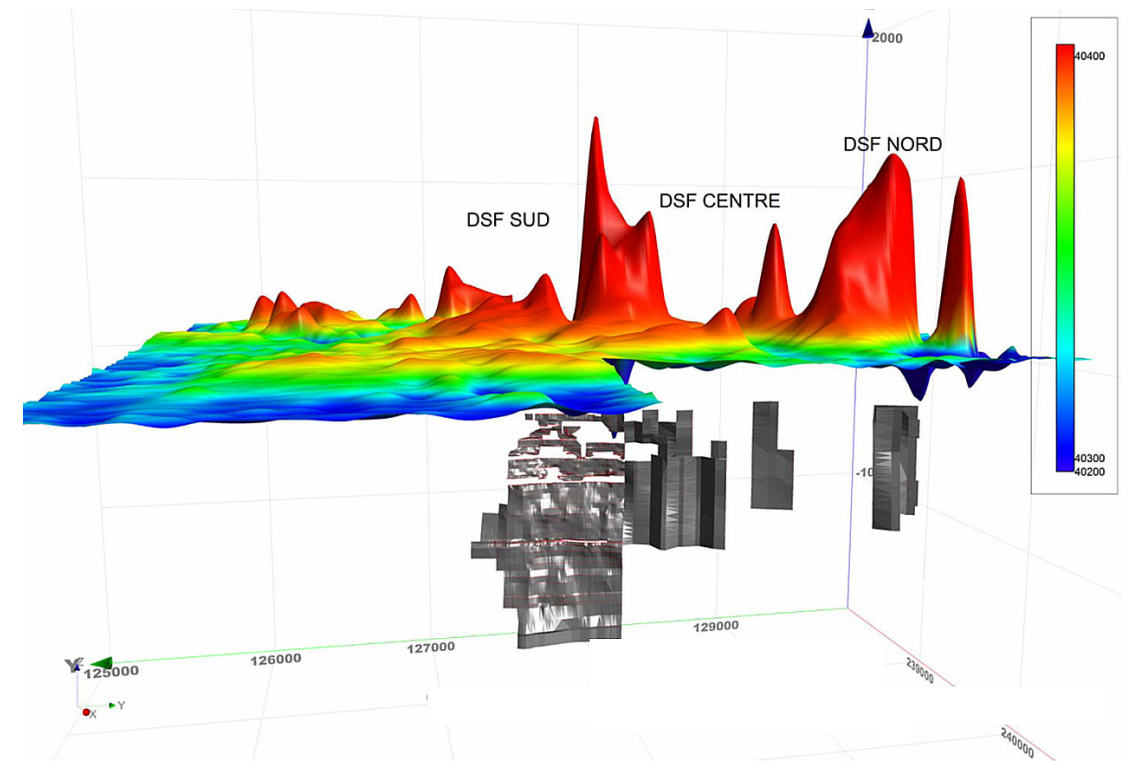

Fig. 8.-Vue en perspective 3D du gisement de Draa Sfar et des anomalies magnétiques, champ total.

\section{Caractéristiques du champ magnétique}

Sur le secteur de Draa Sfar (latitude $31^{\circ} 43^{\prime}$, longitude $\left.-8^{\circ} 8^{\prime} \mathrm{W}\right)$, l'inclinaison du champ magnétique est de l'ordre de $43.2^{\circ} \mathrm{N}$ et sa déclinaison d'environ $3.5^{\circ} \mathrm{W}$. Ces paramètres ont une incidence directe sur l'allure de l'anomalie magnétique en surface, tout comme la morphologie de la source anomale. La réduction au pôle est généralement utilisée pour pallier au problème que pose la variation de l'inclinaison et de la déclinaison magnétique lorsqu'on s'éloigne des pôles magnétiques. Sauf contre indication, ce type de présentation facilite l'analyse des résultats magnétiques, les maximums magnétiques étant situés à l'aplomb des sources anomales.

\section{Modélisation/inversion des résultats magnétiques}

Les minéralisations exploitées à Draa Sfar sont polymétalliques $(\mathrm{Pb}, \mathrm{Zn}, \mathrm{Cu})$ riches en pyrrhotite et reconnues le long d'un mince horizon à sulfures à fort pendage vers l'Est dont la puissance excède rarement 30 mètres. Sa continuité latérale est de l'ordre de 2 kilomètres et sa direction NL16 ${ }^{\circ}$. Le gisement est subdivisé en trois compartiments appelés Draa Sfar Sud, Draa Sfar Centre et Draa Sfar Nord.

Les lentilles à sulfures riches en pyrrhotite sont soulignées en surface par une anomalie magnétique dont l'amplitude maximale est d'environ 450nt (fig. $7 \&$ 8). La couverture magnétique au sol au

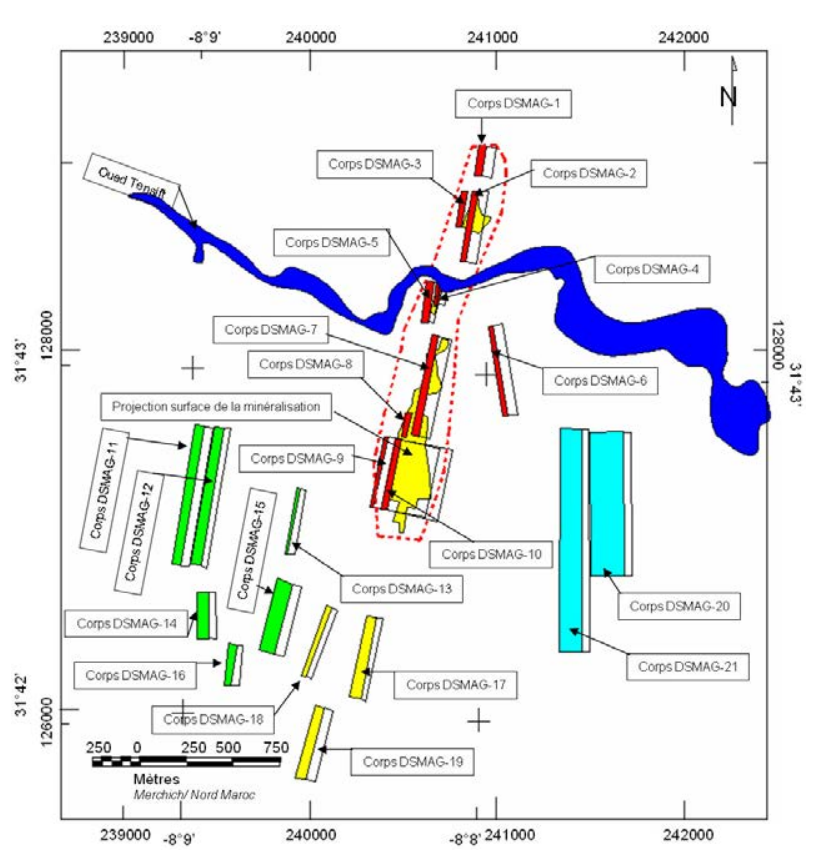

Fig. 9.-Vue en plan des corps magnétiques modélisés.

niveau du gisement a été réalisée suivant un réseau de profils E-O espacés aux 100 mètres avec un point de mesure tous les $12.5 \mathrm{~m}$ ou 25 mètres. Les lacunes dans la couverture magnétique sont relevées au niveau du village de Tazakourt.

Les principales informations au niveau de la mine, concernant les lentilles minéralisées et les 


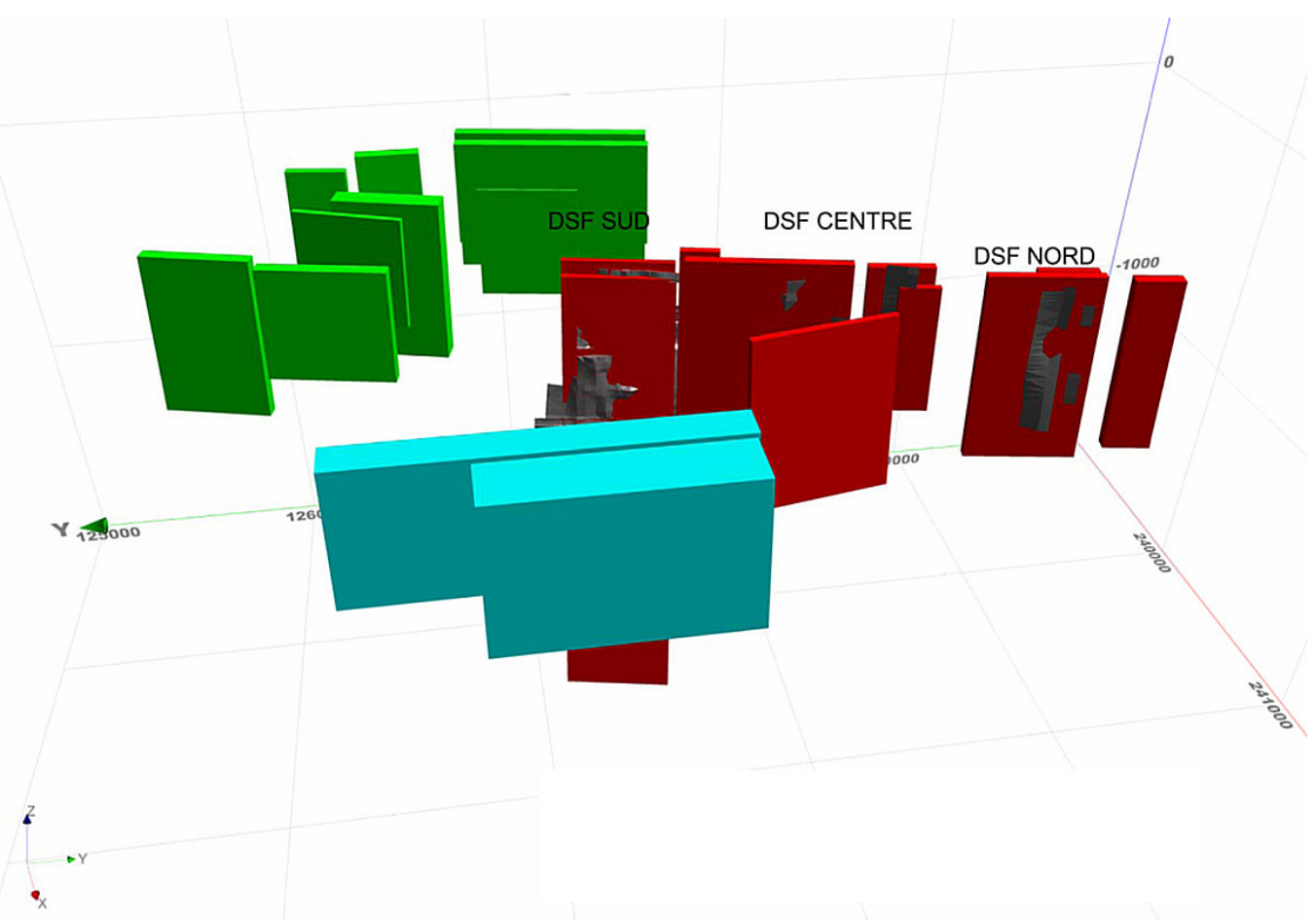

Fig. 10.- Vue en perspective 3D des corps magnétiques modélisés.

lithologies figurant sur le modèle géologique 3D du gisement, ont été transmises en format DXF 3D. Ces fichiers ont ensuite été importés dans le logiciel de modélisation/inversion Model Vision Pro 3D. La base de données de susceptibilité magnétique et de densité issues des mesures effectuées sur les carottes de sondage ainsi que sur des échantillons recueillis en surface, a été importé aussi dans le logiciel Model Vision Pro 3D. Chaque unité géologique a été caractérisée par sa susceptibilité magnétique et sa densité. Ensuite, la réponse magnétique et gravimétrique du gisement et des lithologies environnantes en fonction des propriétés physiques connues a été calculée par le logiciel. L'analyse des résultas magnétiques a ensuite été complétée profil par profil avec le logiciel Profile Analysis. Les limites des différents corps ont été modifiées jusqu'à l'obtention du meilleur ajustement possible entre la courbe de la réponse mesurées et la courbe de la réponse théorique calculée par le logiciel. La localisation en plan et en perspective 3D des corps modélisés est illustrée au niveau des figures 9 et 10. La réponse théorique en surface de ces corps a aussi été simulée, puis comparée à la signature magnétique au sol du secteur de Draa Sfar. La similitude des réponses

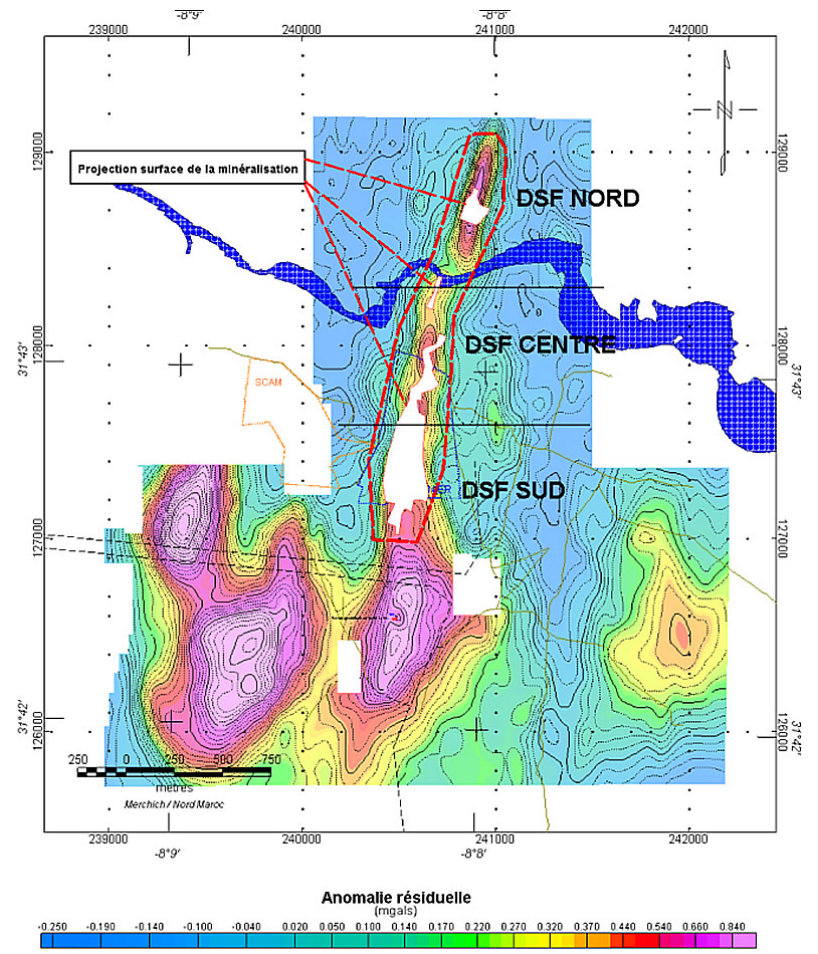

Fig. 11.- Vue en plan du levé gravimétrique, anomalie de Bouger résiduelle. 


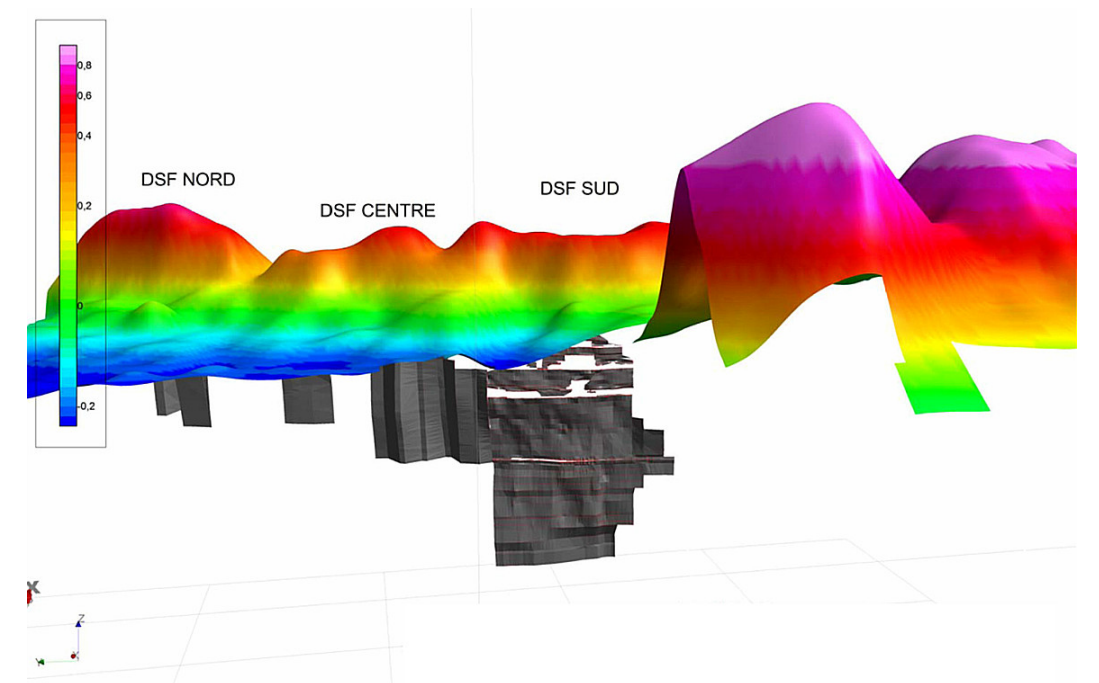

Fig. 12.-Vue en perspective 3D du gisement de Draa Sfar et des anomalies gravimétriques résiduelles.

obtenues rend confiant quant à la solution retenue pour expliquer ces différentes anomalies.

Sur la base de leur susceptibilité magnétique et/ou des considérations géologiques, les corps magnétiques ont finalement été subdivisés en quatre groupes principaux :

- Groupe A (Corps DSMAG-1 à DSMAG-10): Pour l'essentiel ces corps à forte susceptibilités magnétiques sont à mettre en relation avec les minéralisations riches en pyrrhotite associées au gisement.

- Groupe B (Corps DSMAG-11 à DSMAG-16): Leur origine serait attribuable au gabbro dont la présence est connue à l'ouest de Draa Sfar au voisinage des corps DSMAG-11 et DSMAG-12. Une susceptibilité moyenne de l'ordre de 0.004 SI leur est associée, en accord avec les mesures de terrain.

- Groupe C (Corps DSMAG-17 à DSMAG19): La susceptibilité magnétique de ces trois corps est légèrement inférieure à celle moyenne des gabbros et ceux-ci sont situés dans le prolongement sud-ouest du niveau porteur de Draa Sfar. L'examen $\mathrm{du} \log \mathrm{du}$ sondage DS-57 visant l'explication de cette anomalie a intersecté un gabbro identifié DSMAG-18.

- Groupe D (Corps DSMAG-20 et DSMAG21): Ils sont l'expression probable d'une lithologie à faible susceptibilité magnétique recoupée par les sondages DS134 et DS136. Ce sont des métapélites à dissémination de sulfures.

\section{Modélisation/inversion des résultats gravimétriques}

La campagne gravimétrique sur le secteur du gisement a été réalisée suivant un réseau de profils est-ouest espacés aux 100 mètres avec un point de mesure tous les 12.5 ou 25 mètres. La densité moyenne de $2.5 \mathrm{~g} / \mathrm{cm} 3$ a été utilisée lors du calcul de l'anomalie de Bouguer. Les résultats de ce levé sont illustrés en plan et en perspective 3D au niveau des figures 11 et 12 . On retiendra plus spécifiquement que les minéralisations du gisement sont signalées par des anomalies dont l'amplitude oscille entre 0.4 et $1.0 \mathrm{mgal}$, lesquelles se superposent relativement bien aux anomalies magnétiques.

La solution retenue pour expliquer les résultats magnétiques sur le secteur du gisement a donc été utilisée pour initier le travail de modélisation/inversion des données gravimétriques. Cette façon de procéder suppose l'origine de plusieurs anomalies en totalité ou en partie attribuables à la présence d'un même corps dense et magnétique, ce qui devrait normalement être le cas des minéralisations sulfurées de Draa Sfar. En dehors du gisement, ce genre d'approche n'a pas été retenu car les corrélations d'anomalies magnétiques et gravimétriques sont moins convaincantes. L'analyse des résultats a ensuite été réalisée profil par profil en supposant la densité des corps peu variable, là où l'information était disponible. La localisation, en plan et en perspective 3D, des différents corps gravimétriques 


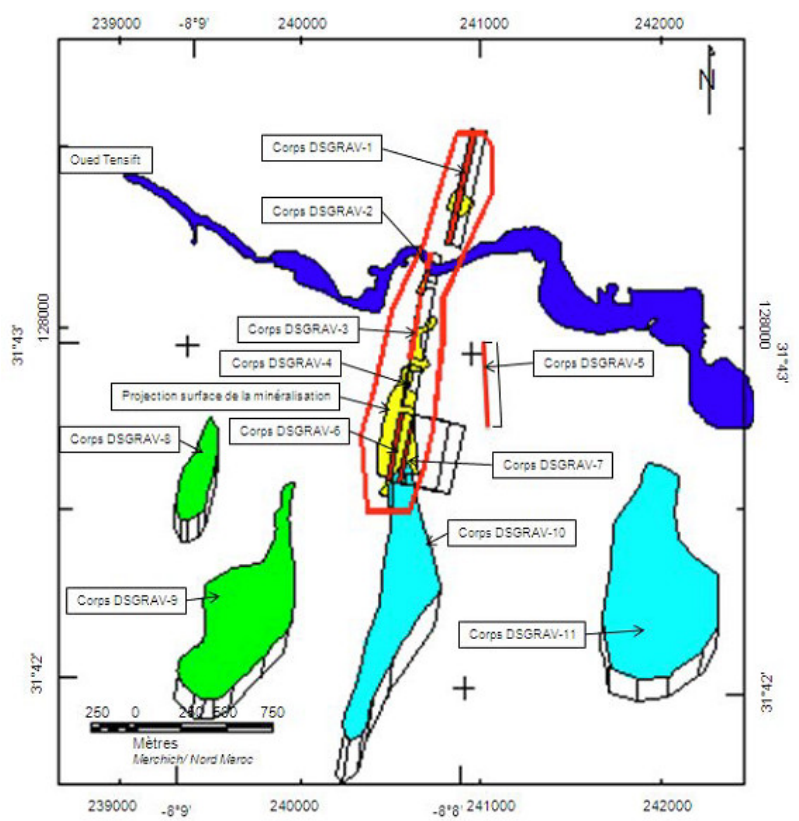

Fig. 13.- Vue en plan des corps gravimétriques modélisés.

modélisés est illustrée au niveau des figures 13 et 14. La réponse simulée en surface du modèle gravimétrique se compare de façon convaincante à la signature gravimétrique mesurée au sol du secteur de la mine. En fonction de leur densité et/ou d'informations géologiques, les corps gravimétriques pourraient être de quatre origines distinctes, à savoir;

- Groupe A (Corps DSGRAV-1 à DSGRAV-7) : Ces corps denses sont induits par des minéralisations sulfurées et leur association avec des corps magnétiques est totale ou partielle.

- Groupe B (Corps DSGRAV-8 et DSGRAV-9) : Le corps DSGRAV-8 est localisé dans les limites d'un affleurement de gabbro et son origine, ainsi que celle du corps DSGRAV-9, est attribuable à cette formation à la fois dense et magnétique. La corrélation des corps magnétiques et gravimétriques sur ce secteur est cependant plus ou moins convaincante ce qui pourrait être lié à des hétérogénéités dans la composition du gabbro.

- Groupe C (Corps DSGRAV-10): Ce groupe met en relief, au moins partiellement, les unités volcanosédimentaires connues au sud de la mine. La signature gravimétrique de ces mêmes unités, également présentes au sud-est et à l'est du gisement, est toutefois nettement moins contrastée, ce qui demeure à expliquer.
- Groupe D (Corps DSGRAV-11): on origine est vraisemblablement lithologique, mais non confirmée pour le moment, tout le secteur étant sous couverture.

\section{Compilation et perspective de développement}

\section{Draa Sfar Sud}

Les anomalies magnétiques et gravimétriques qui marquent la partie sud du gisement sont de plus faible amplitude mais de grande longueur d'onde. Ces observations suggèrent un net approfondissement du sommet des minéralisations vers le sud mais aussi, une certaine continuité verticale du niveau minéralisé. Sur ce dernier point il est important de mentionner que le seuil de détection magnétique ou gravimétrique des minéralisations de Draa Sfar est de l'ordre de 500 à 600 mètres, considérant la morphologie des corps minéralisés et leurs propriétés physiques. Au delà de cette profondeur, l'analyse des résultats devient beaucoup plus complexe.

La carte d'interprétation des modèles géologique et géophysique montre que la minéralisation serait limitée vers le sud par une faille E-W à pendage sud, identifiée F6 d'où la nécessité de continuer le traçage du niveau-640 vers le sud au-delà de la zone mince intersecté sur la coupe DSC245S qui correspond à un haut fond.

\section{Draa Sfar Centre}

Le segment sud du bloc centre nous apparaît nettement plus favorable, plus spécifiquement entre 100 et 500 mètres de profondeur verticale. Vers le nord, en s'approchant de l'oued Tensift, la réponse du corps minéralisé apparaît plus diffuse, suggérant un appauvrissement en sulfures alors que son évolution semble affectée par de nombreuses failles.

Le modèle géologique montre l'existence d'une rupture de pente de part et d'autre du haut fond à partir du niveau 520. Ceci, donne un grand espoir pour l'aval du bloc Central non reconnu. D'où la nécessité de continuer le traçage du N-640 en arrêt sur la coupe DSC210N jusqu'à la coupe DSC1330N avec la réalisation des sondages avals bloc Central à partir du niveau 640. 


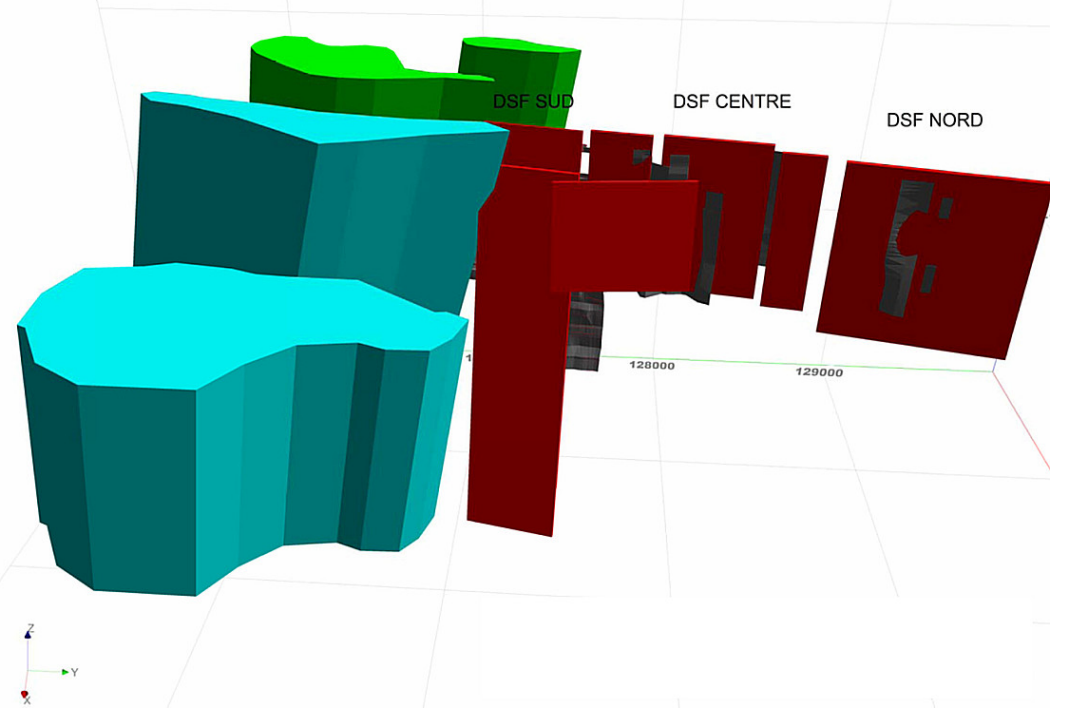

Fig. 14.-Vue en perspective 3D des corps gravimétriques modélisés.

L'anomalie magnétique (DSMAG-6) et gravimétrique (DSGRAV-5) située à l'est du gisement représente, la meilleure cible en dehors du gisement (fig. 7).

\section{Draa Sfar Nord}

D'après les résultats de modélisation, l'essentiel de la réponse anomale serait attribuable à plusieurs corps métriques situés entre 25 et 500 mètres de profondeur verticale. Le potentiel de ce secteur demeure donc intéressant et mériterait un réexamen des données existantes. La continuité aval du niveau nord à des profondeurs supérieures à 500 mètres n'a pu être démontrée voire serait moins probable qu'au niveau de Draa Sfar Centre et surtout Sud.

\section{Conclusions}

Les modèles géologiques et géophysiques tridimensionnels présentés dans cet article ont été construits sur la base des informations géologiques et géophysiques actuellement disponibles. Les solutions retenues sont jugées satisfaisantes, dans la mesure où le calcul de la réponse théorique en surface des corps magnétiques ou gravimétriques permet d'obtenir une image relativement conforme à celles observées suite aux levés au sol. Le modèle est interactif, les corps modélisés ne sont pas figés et pourront être modifiés en fonction de l'ajout de nouveaux résultats de sondages ou pour attester de certaines hypothèses géologiques. Les ressources hypothétiques des cibles d'explorations mises en évidence au voisinage immédiat du gisement sont estimées à $10 \mathrm{Mt}$ à $5 \% \mathrm{Zn}$.

L'exécution des travaux d'exploration tactique, basés sur la méthodologie du modèle géologique et géophysique tridimensionnel des différents indices miniers reconnus dans les Jebilet centrales tel qu'El Mena et Draa Tréfani, permettra de découvrir de nouveau corps minéralisés cachés et d'assurer ainsi le développement minier et économique de la région des Jebilet.

\section{Références}

Aarab, E.M. (1995). Genèse et différentiation d'un magma tholéiitique en domaine extensif intracontinental: l'exemple du magmatisme pré-orogénique des Jebilet (Maroc, Hercynien). Thèse de docteur d'état. Université Cadi ayyad Marrakech, 253 pp.

Belkabir, A.; Gibson, H.; Marcoux, E.; Lenz, D. \& Rziki, S. (2008). Geology and Wall-Rock Alteration at the Hercynian Draa Sfar $\mathrm{Zn}-\mathrm{Pb}-\mathrm{Cu}$ deposit, Morocco. Ore Geology Reviews, 33: 280-306. doi:10.1016/j.oregeorev.2007.03.001

Ben aissi, L.; El Boukhari, A.; Hibti, M.; Harfi, M.; Maacha, L. \& Zinbi, Y. (2005). Altération hydrothermale et déformation ductile des roches volcaniques acides associées au gisement de Draa Sfar Jebilet 
centrales, Maroc. Estudios géologicos, 61: 147-160. doi:10.3989/egeol.05613-652

Ben aissi, L. (2001). Gisement de Draa Sfar: Etude géologique et métallogénique des minéralisations et des altérations hydrothermales associées, Jebilet centrales, Maroc. Thèse 3éme cycle, Université Cadi ayyad, Marrakech, 82 pp.

Bernard, A.J.; Maier, O.W. \& Mellal, A. (1988). Aperçu sur les amas sulfurés massifs des hercynides marocaines. Mineralium deposita, 23: 104-114. doi:10.1007/BF00206659

Bédard, K.; Lachance, B.; Kirkwood, D.; Beaudoin, G. \& Pouliot, J. (2006). Développement d'un prototype de requêtes topologiques $3 D$ et application au gîte de $\mathrm{Cu}-\mathrm{Ag}-\mathrm{Pb}-\mathrm{zn}$ de Transfiguration, Canada. Rapport final projet Divex SC20, Université Laval, Québec, $12 \mathrm{pp}$.

Bordonaro, M. (1983). Tectonique et pétrographie du district à pyrrhotite de Kettara (paléozö̈que des Jebilet, Maroc). Thèse 3éme cycle, Université Louis Pasteur, Strasbourg, $132 \mathrm{pp}$.

Dagbert, M. \& Harfi, M. (2002). Ressource estimation for the Draa Sfar South polymetallic deposit. Exploration and Mining Geology, 11: 99-112. doi:10.2113/11.1-4.99

Essaifi, A. (1995). Relations entre magmatisme, déformation et altération hydrothermale, l'exemple des Jebilet Centrales (Hercynien, Maroc). Mémoires Géosciences Rennes, 66: 340.

Essaifi, A.; Potrel, A.; Capdevila, R. \& Lagarde, JL. (2003). Datation U-Pb: âge de mise en place du magmatisme bimodal des Jebilet centrales (chaîne Varisque, Maroc). Comptes Rendus Géoscience, 335: 193203. doi:10.1016/S1631-0713(03)00030-0

Gibson, H.L.; Brunet, S.; Belkabir, A.; Lentz, D. \& Marcoux, E. (2005). The spectrum of Volcano-sédimentary environments hosting volcanogenic massive sulphide deposits of the Visean, Jebilet and Guemassa Terrains, Morocco. Canadian Institute of Mining and Meta- llurgy, Annual Meeting, Toronto, Ontario, Program with abstracts, p.28.

Hibti, M. (2001). Les amas sulfurés des Guemassa et des Jebilet (Meseta Sud Occidentale, Maroc): Témoins de l'hydrothermalisme précoce dans le bassin mésétien. Thèse de Doctorat, Université Cadi Ayyad, Marrakech, $301 \mathrm{pp}$.

Huvelin, P. (1977). Etude géologique et gîtologique du massif hercynien des Jebilet (Maroc occidental). Notes Mémoires Services Géologiques Maroc, 232 bis: 1-307.

Marcoux, E.; Belkabir, A.; Harold, L.G.; David, L. \& Gilles, R. (2008). Draa Sfar, Morocco: a visiéen (331Ma) pyrrhotite-rich, polymetallic volcanogenic massive sulphide deposit in a Hercynian sedimentdominant terrane. Ore Geology Reviews, 33: 307-328. doi:10.1016/j.oregeorev.2007.03.004

Moreno, C.; Alansari, A.; Sáez, R.; Almodóvar, G.; González, F.; Toscano, M.; Velasco, F.; Playford, G.; Bajddi, A.; Rziki, S. \& Zouhair, M. (2008). Age and depositional environment of the Draa Sfar massive sulphide deposit, Morocco. Mineralium deposita, 43: 891-911. doi:10.1007/s00126-008-0199-x

Rziki, S. (2006). Contribution à l'étude géologique du gisement polymétallique de Draa Sfar, Jebilet centrales, Maroc. Mémoire de fin d'études supérieures spécialisées, Université Cadi ayyad, Faculté des sciences et technique Guéliz, Marrakech, 108 pp.

Souaré, A.T. (1988). Contribution à l'étude des amas sulfurés du district des Jebilet centrales et de leurs altérations supergènes, (chapeau de fer) comparaison avec les minéralisations sulfurées d'Agouim (Haut Atlas, Maroc). Thèse de $3^{\circ}$ cycle. Université Cadi ayyad, Marrakech, 273pp.

Recibido el 7 de julio de 2010 Aceptado el 22 de diciembre de 2010 Publicado online el 7 de octubre de 2011 